\title{
Doubling the number of DBVs and a closer look at their Instability Strip
}

\author{
A. Nitta, ${ }^{1,2}$ S. J. Kleinman, ${ }^{2}$ J. Krzesinski, ${ }^{3}$ T. S. Metcalfe, ${ }^{4}$ A. Mukadam, ${ }^{5}$ F. Mullally, ${ }^{6}$ \\ R. E. Nather, ${ }^{6}$ D. J. Sullivan, ${ }^{7}$ S. E. Thompson, ${ }^{8}$ D. E. Winget, ${ }^{6}$ M. A. Wood ${ }^{9}$ \\ ${ }^{1}$ Gemini Observatory, 670 N A'ohoku PI., Hilo, HI 96720 USA \\ ${ }^{2}$ Subaru Telescope, 650 N A'ohoku PI., Hilo HI 96720 USA \\ ${ }^{3}$ Mt. Suhora Observatory, Cracow Pedagogical University, ul. Podchorazych 2, 30-084 Cracow, Poland \\ ${ }^{4}$ High Altitude Obs., National Center for Atmospheric Research, P.O. Box 3000, Boulder CO 80307, USA \\ ${ }^{5}$ Dept. of Astronomy, Univ. of Washington, 3910 15th Ave NE, Seattle WA 98195 \\ ${ }^{6}$ Astronomy Dept. \& McDonald Observatory, University of Texas at Austin, Austin, TX \\ ${ }^{7}$ School of Chemical \& Physical Sciences, Victoria University of Wellington, Wellington, NZ \\ ${ }^{8}$ Dept. of Physics, Colorado College, 14 E. Cache La Poudre, Colorado Springs, CO 80903 USA \\ ${ }^{9}$ Dept. of Physics \& Space Science, SARA Obs., Florida Institute of Technology, Melbourne, FL, USA
}

\begin{abstract}
Prior to the Sloan Digital Sky Survey (SDSS), there were only nine known DBVs compared to 35 DAVs. The latest SDSS DR4 White Dwarf Catalogue (Eisenstein et al. 2006) has quadrupled the number of known white dwarf stars. We have been searching for new DBVs from the SDSS catalogue. Increased numbers of DBVs will help us better understand the structure and evolution of DBs, the nature of their instability strip as well as plasmon neutrino processes (Winget et al. 2004). We searched for DBV candidates using effective temperatures and surface gravities determined by fitting SDSS spectra with Koester's atmosphere models. We then obtained time-series photometric data on those with fit temperatures near those of the known pulsators. So far we have discovered 8 new DBVs, nearly doubling the number of previously known DBVs. With increased numbers of DBVs, we will be able to better characterize the instability strip, but, we also need more precise determinations of the temperatures and surface gravities via better signal to noise spectra and better lower limits for the observed non-variables. This effort is ongoing.
\end{abstract}

Acknowledgments. Funding for the SDSS and SDSS-II has been provided by the Alfred P. Sloan Foundation, the Participating Institutions, the National Science Foundation, the U.S. Department of Energy, the National Aeronautics and Space Administration, the Japanese Monbukagakusho, the Max Planck Society, and the Higher Education Funding Council for England. The SDSS Web Site is http://www.sdss.org/.

\section{References}

Beauchamp A., Wesemael F., Bergeron P., et al., 1999, ApJ, 516, 887

Eisenstein D. J., Liebert J., Harris H. C., et al., 2006, ApJS, 167, 40

Winget D. E., Sullivan D. J., Metcalfe T. S., Kawaler S. D., Montgomery M. H., 2004, ApJ, 602, L109 


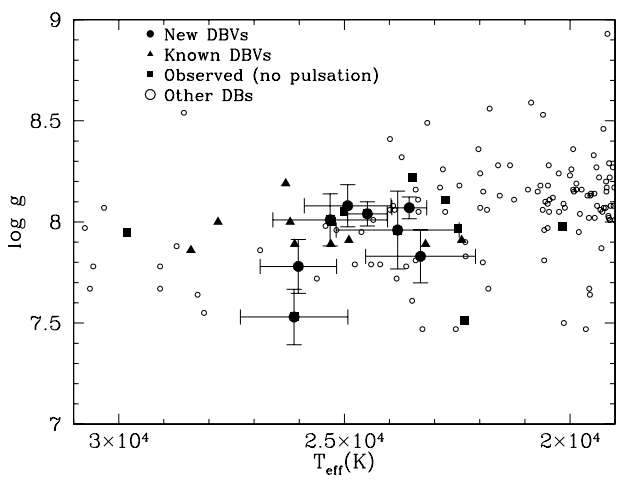

Figure 1: Effective temperatures and surface gravities of the SDSS DBs (Eisenstein et al. 2006) around the DBV instability strip, along with the previously known DBVs with their physical parameters taken from Beauchamp et al. (1999). Solid dots show the new DBVs and triangles the previously-known DBVs. Squares show the DBs which did not show any variability and hollow circles show the rest of the DBs in the SDSS DR4 WD catalogue. Most of the DBs which we did not see any variability so far have such high amplitude limits that we cannot tell if they are truly non-pulsators or not. To characterize the instability strip better, we need better determinations of the physical parameters (from better signal-to-noise spectra) and better variability amplitude limits (1 mma or better). We found no pulsator hotter than EC20058 and hence the best chance of determining the neutrino production rates still lies with this star.

Table 1: Results of our work so far. The top section of the table shows the objects that showed variability during at least one observation. Separated by a double vertical line, the second half of the table shows the objects which did not yet show variability. In the status section, we noted the objects which showed variability by "DBV". For the objects we have not seen variability of, we put the amplitude limit in the status section. The objects we have only observed once are noted by (1). Beating of multiple modes and amplitude modulation can make a pulsator appear as a non-pulsator. Therefore, we aim to observe each object at least two separate times, including new pulsators to ensure we have found a real pulsator.

\begin{tabular}{lllllll}
\hline Object (SDSS J) & $\mathrm{g}[\mathrm{mag}]$ & $T_{\text {eff }}$ & $\sigma_{\text {Teff }}$ & $\operatorname{logg}$ & $\sigma_{\text {logg }}$ & Status \\
\hline $034153.03-054905.8$ & 18.113 & 24490 & 440 & 8.04 & 0.060 & DBV \\
$094749.40+015501.8$ & 20.034 & 23819 & 1362 & 7.96 & 0.192 & DBV $(1)$ \\
$140814.63+003838.9$ & 18.981 & 25314 & 1271 & 8.01 & 0.129 & DBV $(1)$ \\
$125759.03-021313.3$ & 19.050 & 26114 & 1191 & 7.53 & 0.137 & DBV \\
$104318.45+415412.5$ & 19.041 & 26020 & 846 & 7.78 & 0.133 & DBV \\
$122314.25+435009.1$ & 18.838 & 23312 & 1218 & 7.83 & 0.131 & DBV(1) \\
$130516.51+405640.8$ & 17.389 & 23562 & 386 & 8.07 & 0.054 & DBV \\
$130742.43+622956.8$ & 18.710 & 24926 & 962 & 8.08 & 0.104 & DBV(1) \\
\hline \hline $001529.74+010521.3$ & 18.711 & 35974 & 899 & 8.00 & 0.129 & $7.2(1)$ \\
$085950.29-000339.6$ & 20.022 & 25289 & 2588 & 8.00 & 0.289 & $11.8(1)$ \\
$090409.03+012740.9$ & 17.850 & 22480 & 521 & 7.97 & 0.060 & 3.5 \\
$090456.11+525029.8$ & 18.665 & 36708 & 615 & 7.94 & 0.087 & $9.0(1)$ \\
$092200.97+000834.3$ & 18.450 & 22754 & 729 & 8.11 & 0.070 & $6.8(1)$ \\
$095256.68+015407.6$ & 17.292 & 32600 & 295 & 8.15 & 0.038 & $4.3(1)$ \\
$095649.55+010812.4$ & 20.361 & 17143 & 915 & 7.32 & 0.240 & $11.5(1)$ \\
$101131.88+050729.3$ & 18.841 & 24767 & 937 & 7.79 & 0.106 & $8.1(1)$ \\
$101502.95+464835.3$ & 18.433 & 23355 & 552 & 8.05 & 0.068 & $6.2(1)$ \\
$105929.60+554039.2$ & 18.458 & 24877 & 553 & 8.10 & 0.099 & $7.6(1)$ \\
$122241.27-003614.4$ & 17.947 & 23497 & 624 & 8.22 & 0.061 & $4.1(1)$ \\
$133215.93+640656.2$ & 18.285 & 20176 & 751 & 7.98 & 0.076 & $8.7(1)$ \\
$135610.32-002230.6$ & 19.230 & 17033 & 265 & 8.00 & 0.155 & $11.1(1)$ \\
$141258.17+045602.2$ & 17.191 & 29822 & 318 & 7.95 & 0.040 & 2.6 \\
$231324.24-001636.9$ & 19.632 & 22331 & 3809 & 7.51 & 0.309 & $16.9(1)$ \\
$235322.16+002653.8$ & 19.594 & 25000 & 1649 & 8.05 & 0.186 & $11.2(1)$ \\
\hline & & & & & &
\end{tabular}

